A. K. Dupree and A. O. Benz, Eds.

\title{
Spectral Analysis of Stars on Planet-Search Surveys
}

\author{
Debra Fischer \\ San Francisco State University, 1600 Holloway, Dept Physics and \\ Astronomy, San Francisco, CA 94132
}

Jeff A. Valenti

Space Telescope Science Institute, Baltimore, MD 21218

\author{
Geoff Marcy \\ University of California, Berkeley, Berkeley, CA 94720
}

\begin{abstract}
.
We present spectroscopic analysis of $\sim 1000$ stars on the Lick, Keck and AAT planet search projects. This analysis provides a quantitative, and unbiased correlation between metallicity and the rate of occurrence of detected gas giant planets with orbital periods shorter than three years. As stellar metallicity increases, the occurrence of planets increases. Stars with $[\mathrm{Fe} / \mathrm{H}]$ that is one third of solar only have gas giants detected $\sim 3 \%$ of the time. Stars with solar metallicity have a planet occurrence rate of $5-10 \%$. The occurrence of gas giant planets rises to $20 \%$ in stars with a metallicity that is three times solar.

At issue is whether the quantitative dependence of planet occurrence on metallicity is primarily an initial condition, or a by-product of accretion of gas-depleted material onto the convective zone of the star. Accretion could be distinguished as the underlying mechanism for enhanced metallicity if: 1) planet-bearing F-type stars with thinner convective envelopes show a higher mean metallicity than planet-bearing G- or K-type stars, or 2) planet-bearing sub-giants with diluted convective zones showed statistically lower metallicity than their main sequence counterparts
\end{abstract}

\section{Introduction}

Since 1995, more than 100 extrasolar planets have been discovered by Doppler surveys (for updates see http://exoplanets.org). The Doppler detection of an extrasolar planet requires velocity measurements over one full orbital period. Thus, there is a gradual harvesting of planets from small to wider orbital separations. Nearly all of the planets detected before 1999 reside in orbits with semi-major axes less than $1 \mathrm{AU}$, while now most detected planets have semimajor axes beyond $1 \mathrm{AU}$. 
Around F and $\mathrm{G}$ stars, gas giant planets are expected to form outside of a 3 AU radius, where condensation and accretion of ice grains can occur. So, the discovery of Jovian mass planets closer than 1 AU prompted a cascade of theories on orbital migration, planet-planet gravitational interactions and planet-disk tidal interactions (Goldreich \& Tremaine 1980, Lin, Bodenheimer \& Richardson 1996, Rasio \& Ford 1996, Weidenschilling and Marzari 1996, Levison et al. 1998, Bryden et al. 1999, Murray \& Holman 2001, Chiang et al. 2002, Malhotra 2002, Ford et al., 2003). Implicit in the concept of orbital migration is the expectation that not all migrating planets and planetesimals will park in stable orbits; some gas-depleted material will fall into the star and mix in the convective zone, possibly enriching the abundance of refractory elements (Lin 1997). Even in our own solar system, with a presumably gentler early dynamical history, the fossil record of a cratered surface on Mercury supports this concept of late stage accretion.

In parallel with the discovery of the first few extrasolar planets and with the emerging theories of orbital migration, planet-bearing stars were observed to have metallicities higher than the average field star (Gonzalez 1997, Lin 1997, Laughlin \& Adams 1997). The metallicity correlation for planet-bearing stars has persisted and has become the subject of ongoing debate (Laughlin 2000, Pinsonneault et al. 2001, Butler et al. 2000, Santos et al. 2000, Murray \& Chaboyer 2002). At issue is the question of whether the metallicity correlation is primarily an initial condition, or a by-product of the accretion of gas-depleted material. The underlying mechanism for the planet-metallicity correlation bears upon processes of planet formation and bears on the fraction of stars in our galaxy likely to host extrasolar planets.

\section{Spectral Analysis Method}

Spectral analysis was carried out for 1632 spectra of 971 FGK stars in the Lick, Keck and AAT planet search surveys using the spectral synthesis package SME (Valenti \& Piskunov 1996, Valenti \& Fischer 2004). In the analysis, spectral line profiles and continuum for about $200 \AA$ are fit with a synthetic model. Changes in the free parameters (e.g., $T_{\text {eff }},[\mathrm{Fe} / \mathrm{H}], v \sin i, \log g$ ) are driven by a Marquardt minimization of $\chi^{2}$ to match the observed spectrum.

Prior to the analysis, wavelength regions are selected that are free of telluric contamination and common to the spectral formats of the three planet search projects. The initial atomic line data (log $g f$ values, van der Waals broadening coefficients and line wavelengths) were taken from the Vienna Atomic Line Database (VALD, Kupka et al. 1999) and were slightly adjusted to "astrophysical values" by matching spectral lines in a spectral atlas of the Sun at disk center (Wallace, Hinkle, \& Livingston). As a check, the adjusted atomic line data were then used to recover solar parameters $\left(T_{\text {eff }},[\mathrm{Fe} / \mathrm{H}], v \sin i\right)$ from an observation of Vesta.

The analysis made serendipitous use of template spectra from the Doppler planet search stars. The template spectra have a resolution of better than 70000 and signal-to-noise of about 500. Several test iterations of spectral synthesis modeling were run on subsets of a few hundred stars ranging in temperature from $4900 \mathrm{~K}$ to $6400 \mathrm{~K}$ in order to refine wavelength and spectral line selection, 
improve continuum fitting, and derive an empirical measure of macroturbulent velocity as a function of $T_{\text {eff }}$.

\subsection{Uniqueness of the spectral model}

One challenge for spectral synthesis modeling is that different parameters can have similar effects on a spectral line. For example, decreasing the model $T_{\text {eff }}$ will generally make neutral metal lines deeper, but a decrease in the model elemental abundance can compensate. The wide range of excitation potentials and different ionization states of the $\sim 300$ analyzed spectral lines helps to break the degeneracy between $T_{\text {eff }}$ and $[\mathrm{Fe} / \mathrm{H}]$.

A particular challenge has been determination of surface gravity in our stars. Our Monte Carlo simulations show that an error of +0.1 dex in $\log g$ forces an error of up to $+100 \mathrm{~K}$ in $T_{\text {eff }}$, which in turn can result in errors of up to $+0.1 \mathrm{dex}$ in elemental abundances. For the wavelength segments in the analysis presented here, Marquardt minimization did not provide well-constrained values for $\log g$. In Monte Carlo simulations, similar $\chi^{2}$ values were obtained over a range of 0.2 dex in $\log g$. Likewise, when multiple spectra of the same star were analyzed there was unacceptable scatter in $T_{\text {eff }}$ and $[\mathrm{Fe} / \mathrm{H}]$ when $\log g$ was left as a free parameter. Since Hipparcos parallaxes permit the determination of luminosity with high precision, the radius was defined from the luminosity and our $T_{\text {eff }}$. We initially used a mass-luminosity relation to derive stellar mass and fix $\log g$ :

$$
g=g[M(L, R), R(L, T)]=G * M / R^{2}
$$

However, to put our analysis of surface gravity on firmer empirical footing, we have recently added a new wavelength segment that includes the $\mathrm{MgI}$ "b" triplet lines and we are reanalyzing all spectra. For main sequence stars, the derived gravities match those in our previous analysis, but we now recover subgiant surface gravities that are consistent with those of Allende Prieto \& Lambert (1999), who combined Hipparcos-based luminosities and evolutionary tracks.

\subsection{Determining Macroturbulence}

In a synthetic model, rotational velocity $(v \sin i)$, spectrometer resolution, and macroturbulent velocity broaden lines without changing equivalent width. In order to determine $v \sin i$ from the spectral synthesis model, the IP resolution was determined independently by measuring the width of thorium lines. The remaining spectral line broadening is then a combination of $v_{\mathrm{m}} \mathrm{c}$ and $v \sin i$.

Macroturbulent velocity is attributed to large scale convective motions in the stellar photosphere. Thus, macroturbulence is expected to depend on the physical structure of the convective zone or equivalently, on the effective temperature or luminosity of the star. To determine the dependence of $v_{\mathrm{m} \text { c }}$ on $T_{\text {eff }}$, we artificially set $v \sin i$ to zero, absorbing all macroturbulent and rotational broadening into the " $v_{\mathrm{m}} \mathrm{c}$ " parameter. Figure 1 shows the results as a function of $T_{\text {eff }}$. The lower envelope is defined by stars in which macroturbulent broadening dominates rotational broadening. A linear fit to this floor yields the relationship,

$$
v_{m c}=-7.9+T_{\text {eff }} / 500 \text {. }
$$




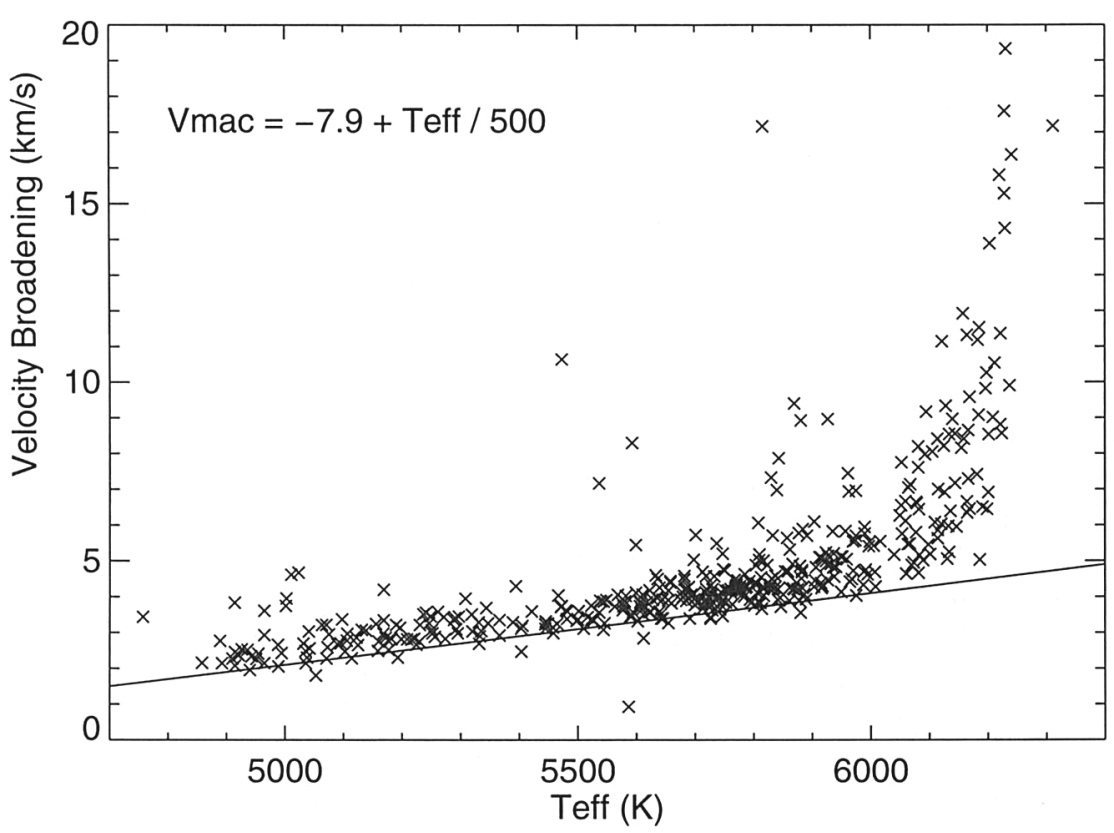

Figure 1. Macrobroadening as a function of $T_{\text {eff }}$. The lower envelope gives macroturbulent velocity as a function of effective temperature.

As the free parameter $T_{\text {eff }}$ and the Kurucz model atmospheres are updated with each Marquardt fitting iteration, the macroturbulent velocity is also updated according to this empirical relation. Because SME runs in batch mode, it is possible to analyze sets of several hundred stars and identify problems in the fits for particular spectral lines as a function of $T_{\text {eff }}$. Once the atomic line parameters and input parameters (such as $v_{\mathrm{m}} \mathrm{c}, v_{m i c}$, IP resolution) are determined, then each spectrum can be analyzed in $1-2$ hours on a Sun Ultra 60.

\subsection{Precision and Accuracy}

The Marquardt fitting algorithm in SME provides an estimate of the formal uncertainty in each free parameter. By fitting hundreds of spectral lines, we typically achieve formal uncertainties of $\pm 30 \mathrm{~K}$ for $T_{\text {eff }}, \pm 1 \mathrm{kms}^{-1}$ for $v \sin i$, and \pm 0.03 dex for $[\mathrm{Fe} / \mathrm{H}]$. A comparison of multiple observations of the same star taken at the same observatory, or observations of the same star taken at multiple observatories, confirms these formal uncertainties. Reduced $\chi^{2}$ for our spectrum fits is typically $\sim 4$ for late $\mathrm{F}$ stars, rising to $\sim 20$ for early $\mathrm{K}$ stars, indicating that formal uncertainties are driven by residual errors in line data, rather than noise in the observed spectrum. As usual with high quality observed spectra, systematic errors probably dominate the formal uncertainties. 

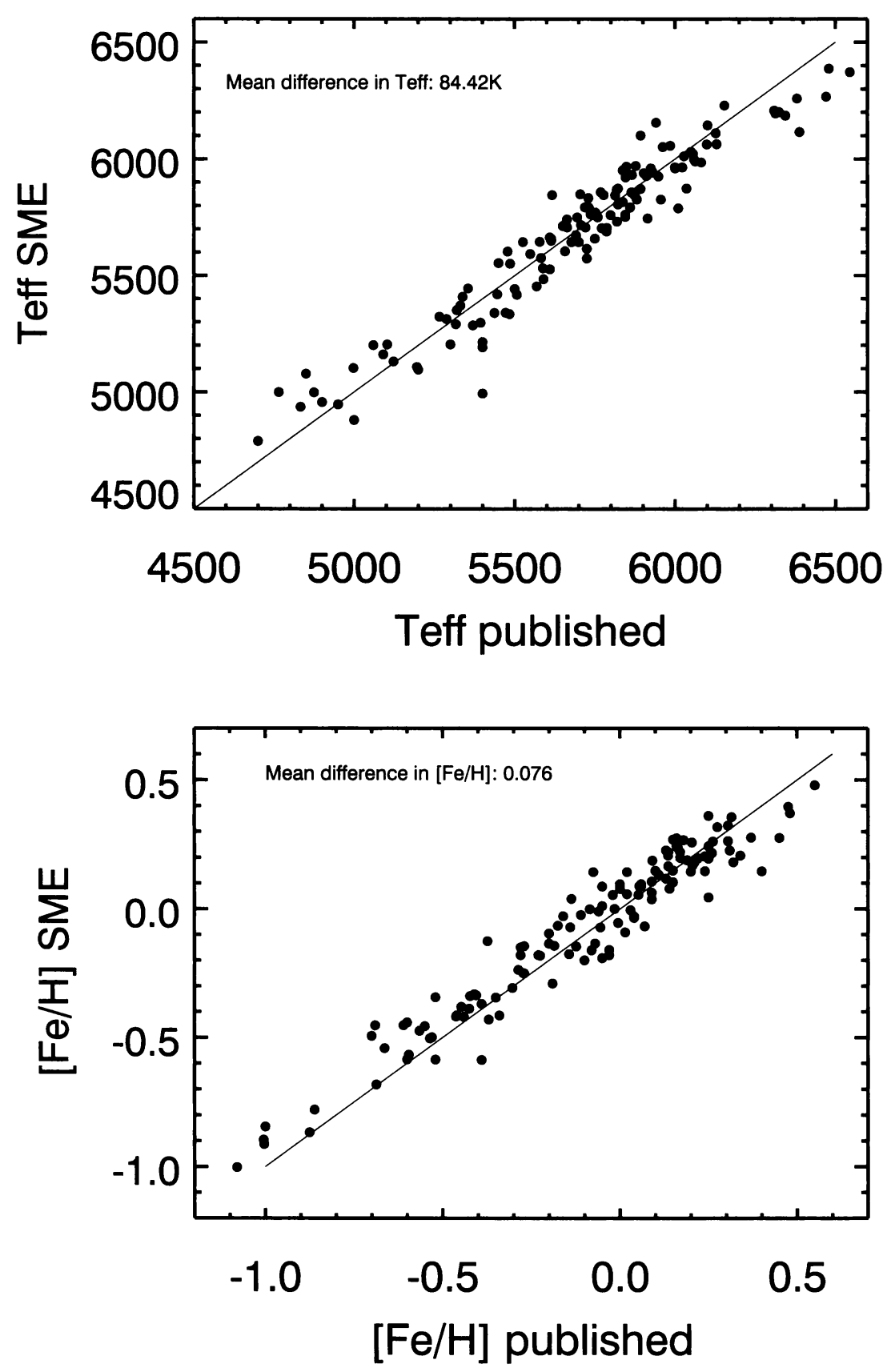

Figure 2. Comparison of SME-derived $T_{\text {eff }}$ and $[\mathrm{Fe} / \mathrm{H}]$ with published values compiled by Cayrel de Strobel. 

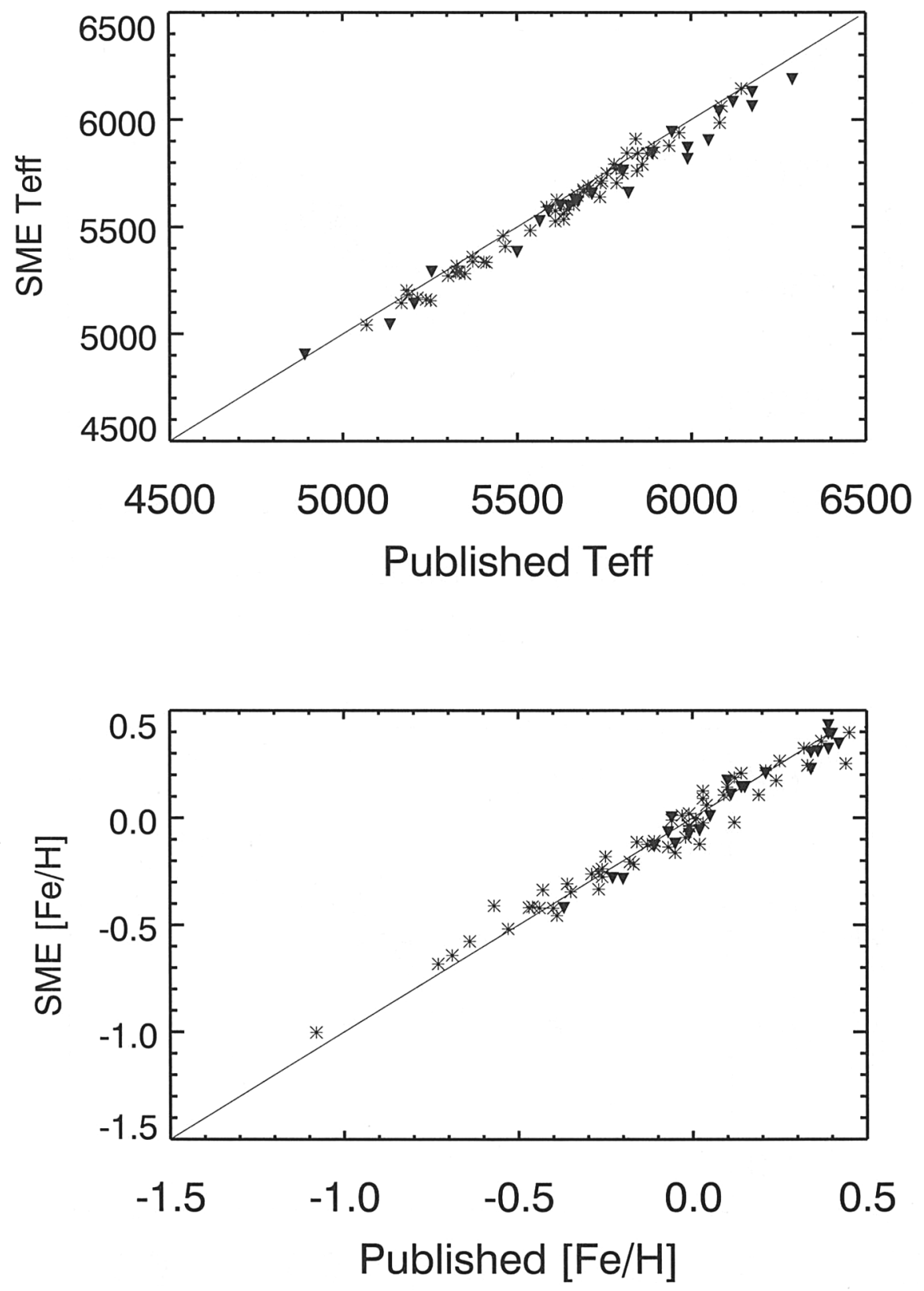

Figure 3. Comparison of SME effective temperatures and metallicities with published values from Fuhrmann (circles) and Santos (filled triangles). Our $T_{\text {eff }}$ are systematically above Fuhrmann by $+33 \mathrm{~K}$ and above Santos by $+58 \mathrm{~K}$. Our $[\mathrm{Fe} / \mathrm{H}]$ agree with scatter of 0.01 and 0.04 dex, respectively. 
To assess the accuracy of our analysis we compare our results to published values compiled by Cayrel de Strobel (2001). This comparison, plotted in Figures $2 \mathrm{a}$ and $2 \mathrm{~b}$, shows RMS agreement to $85 \mathrm{~K}$ in $T_{\text {eff }}$ and 0.08 dex in $[\mathrm{Fe} / \mathrm{H}]$. We also compare our results with the analysis of Fuhrmann (1998) and Santos et al. (2003) in Figure 3 and find agreement to better than $50 \mathrm{~K}$ in $T_{\text {eff }}$ and better than $0.04 \mathrm{dex}$ in $[\mathrm{Fe} / \mathrm{H}]$. The good external agreement at least demonstrates that consistent assumptions and stellar models are being used by various researchers carrying out spectral analysis.

\section{The Metallicity Correlation}

The metallicity correlation was established on the basis of a comparison between planet-bearing stars relative to field stars. Some comparisons use metallicities for field stars determined by a different researcher than the spectroscopic analysis for the planet-bearing stars. Given the concern of systematic differences, it is also important to consider the metallicity of planet-bearing stars relative to all stars in the Doppler planet search program to remove any sample bias.

For stars with $0.5<B-V<1.0$ (late-F to early-K spectral classes), the combined Lick, Keck, and AAT sample is nearly complete out to 15 pc. In Figure 4 we compare the normalized $[\mathrm{Fe} / \mathrm{H}]$ distribution for the 71 stars in this volume-limited subsample with the $[\mathrm{Fe} / \mathrm{H}]$ distribution for stars in the full sample with known planets. There is an obvious offset between these two distributions and a Kolmogorov-Smirnov statistical test sets the probability that these two distributions are drawn from the same parent population at 0.000013 , supporting previous findings (beginning with Gonzalez et al. 1997), that stars with Doppler-detected planets appear to be metal-rich relative to the typical field stars.

Spectral synthesis analysis was carried out for 971 stars in the three Doppler surveys. However, to investigate the correlation between metallicity and the presence of a planet, we restricted our attention to those stars with enough observations to determine whether or not there was planet with $M \sin i>1 \mathrm{M}_{\mathrm{JUP}}$ and an orbital period less than 3 years. This condition on planet detectability was met for 754 stars with at least ten observations spanning a minimum of two years. Indeed, most of these 754 stars had many more than the minimum number of observations spanning a much longer time interval. This detectability requirement means that we do not include in this analysis our abundances determinations for some stars with planets discovered by other groups because formally we do not yet have enough observations to guarantee planet detection.

We then divided this subset of 754 well-observed stars into $[\mathrm{Fe} / \mathrm{H}]$ bins with a bin width of 0.25 dex. In each bin, we determined the fraction of stars with detected extrasolar planetary systems (one or more detected planets) with orbital periods less than 3 years. Figure 5 shows, with Poisson error bars, the fraction of stars in each bin that have detected planets as a function of $[\mathrm{Fe} / \mathrm{H}]$ of the host star. At the top of double bins, the total number of stars is given. These results quantify the metallicity correlation with planet occurrence, overcoming any selection effects or sample bias by including stars with and without detected planets in the analysis. The statistics are free of selection effects and biases and 


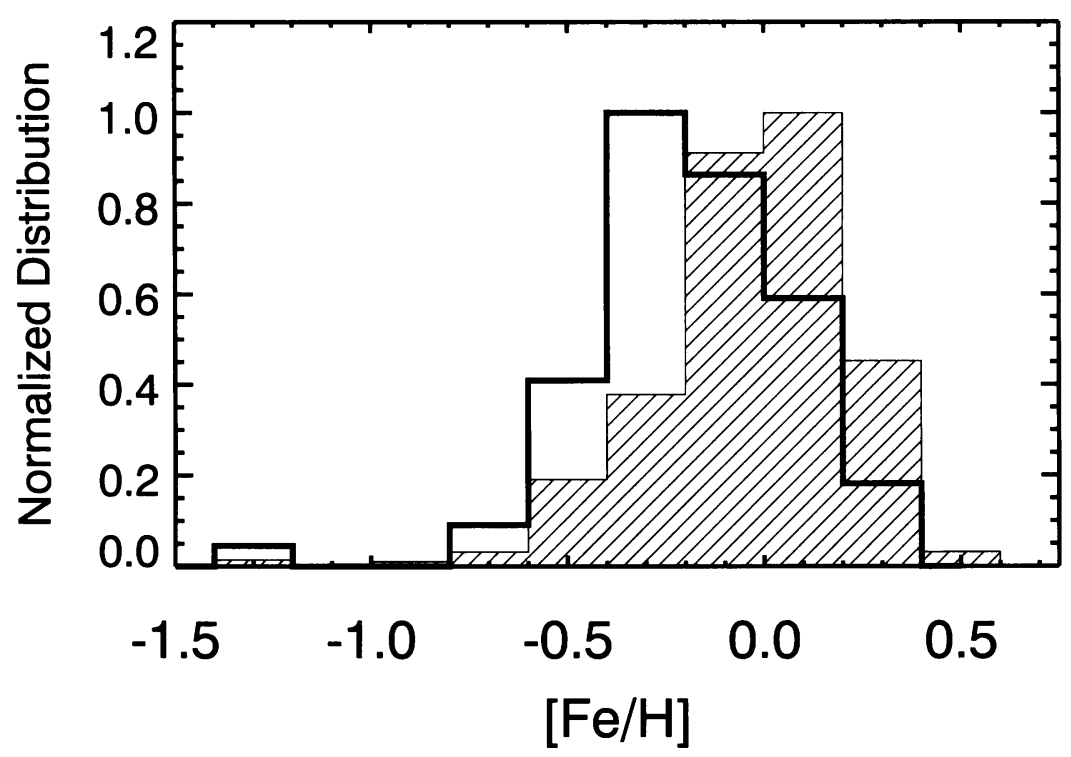

Figure 4. Normalized metallicity distribution for a $15 \mathrm{pc}$ volumelimited subset of our sample (bold outline) and for stars with planets (diagonal stripes).

complete for gas giant planets with orbits shorter than 3 years. Three main conclusions are:

- Between $5-10 \%$ of stars with solar metallicity host Dopplerdetected planets.

- As the stellar metallicity rises toward $[\mathrm{Fe} / \mathrm{H}]=+0.5$, nearly $20 \%$ of the stars are found to have gas giant planets.

- As stellar metallicity drops toward $[\mathrm{Fe} / \mathrm{H}]=-0.5$, the occurrence of detected planets declines to a few percent.

One important question is whether some planets are not detected around lower metallicity stars because their spectra have fewer and shallower spectral lines. If so, our conclusions would be suspect because of an observational bias. To assess the effect of lower metallicity, the median velocity error was calculated for each metallicity bin. While the median velocity precision dropped from 3 $\mathrm{m} \mathrm{s}^{-1}$ for the most metal-rich bin to $4.7 \mathrm{~m} \mathrm{~s}^{-1}$ for the most metal-poor bin, this lower precision is still sufficient to detect a Jupiter-mass planet. Velocity precision does not suffer significant degradation until metallicity drops below $[\mathrm{Fe} / \mathrm{H}] \sim-1.0$. 


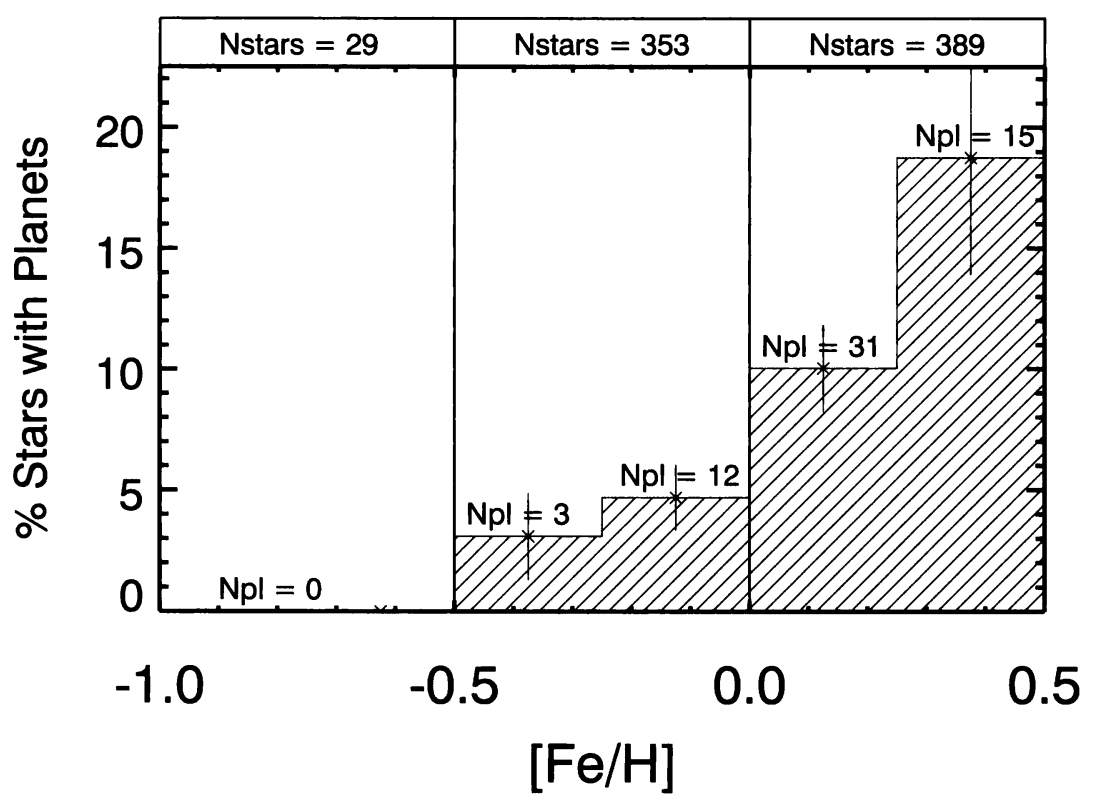

Figure 5. Rate of occurrence of planets as a function of metallicity for 754 stars with at least 2 years of observations and 10 Doppler measurements. These selection criteria allows us to rule in (or out) the presence of a planet with $M \sin i>1 \mathrm{M}_{\mathrm{JUP}}$ in orbits less than 3 years. The occurrence of planets is a sensitive function of metallicity of the host star.

\section{Interpretation}

With this quantification of the metallicity correlation, we consider whether the data can distinguish whether planetary companions enhance stellar metallicity through accretion or whether enhanced metallicity in the parent cloud breeds more planets. We consider three observational tests that could lend strong support to accretion under the assumption that infalling material in late-stage accretion mixes in the convective envelope of the star, emphasizing that none of these trends are observed:

Test 1: A rising upper envelope in $[\mathrm{Fe} / \mathrm{H}]$ distribution as a function of $T_{\text {eff. }}$.

Test 2: A higher $[\mathrm{Fe} / \mathrm{H}]$ for stars with planets in closer orbits relative to those with planets in more distant orbits. 
Test 3: A statistically lower metallicity in planet-bearing subgiants with diluted convective zones.

For the first of these three tests, Pinsonneault, DePoy \& Coffee (2001) have modeled the effect of adding a few Earth masses of heavy elements to the convective zone of a main sequence star and show that independent of the initial stellar metallicity a dramatic rise should be observed for stars with $T_{\text {eff }}>6200$ K. Figure 6 shows that the metallicity distribution for the subset of 754 wellobserved stars at Keck, Lick and the AAT is not consistent with accretion of even a few Earth masses of metals. There is a spread in metallicity at all $T_{\text {eff }}$, however the upper envelope of this distribution is not rising as the convective zone is thinning. If accreted material plunges through, or settles out of the convective zone and into the radiative zone of the star then the metallicity correlation would disappear. To increase the metallicity of the entire interior of the host star (not just the convective zone) by a factor of $\sim 3$ would require accretion of the total metal content of the gas-depleted protoplanetary disk.

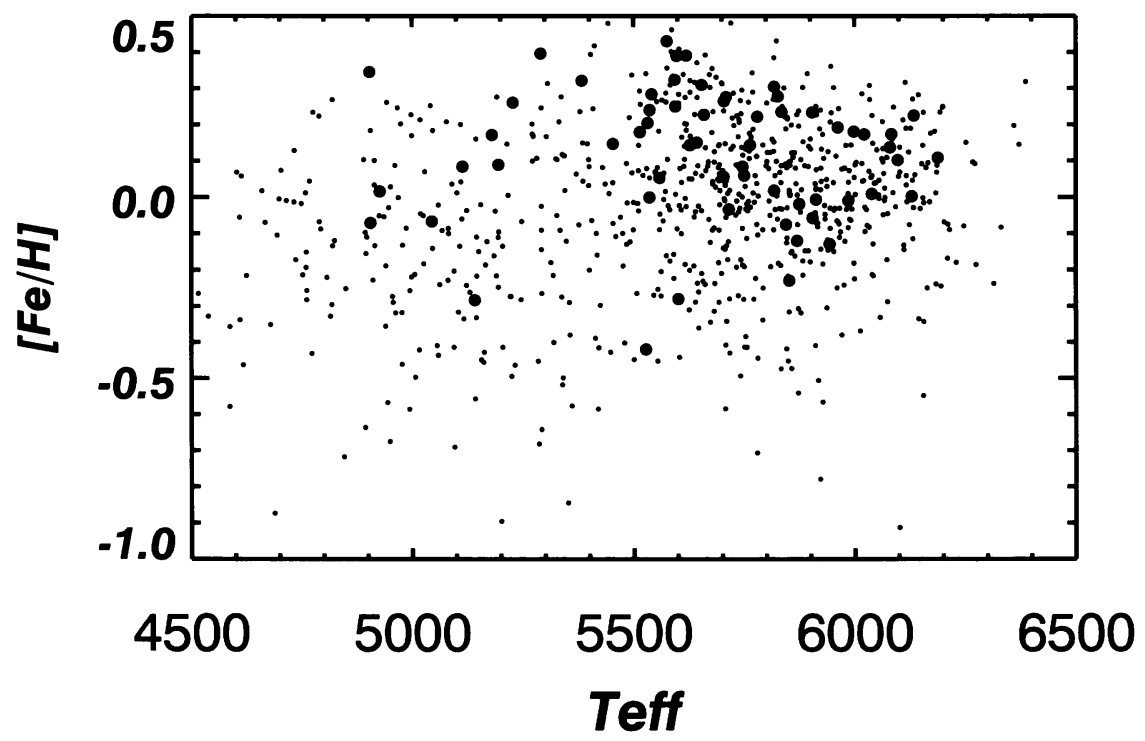

Figure 6. Stellar metallicity as a function of effective temperature. Bold dots indicate stars with planets. No rise in the upper envelope of metallicity is observed as a function of $T_{\text {eff }}$, suggesting that enrichment of the convective zone by accretion did not occur.

The second test is more difficult to quantify. The premise is that gas giant planets in close orbits may be a signature of inward migration, so host stars in such systems may have experienced more accretion because the observed planets 
are a survivors and a signature of inward migration. However, the planetary systems considered here have orbital periods less than 3 years and probably all experienced a measure of inward migration. It will take several more years until solar system architectures are discovered where the planets have not undergone substantial migration.

The subgiants with extrasolar planetary companions in our sample share the same characteristic of high metallicity with main sequence stars. Thus, as the stars evolve and the convective zone deepens, dilution of initial high metallicity in the convective zone of planet-bearing stars is not observed. The stars appear to be metal-rich throughout indicating that the metallicity was high in the original molecular cloud core.

\subsection{Acknowledgments}

We thank Paul Butler and Steve Vogt for the use of their Keck template spectra and for helpful discussions. We thank Greg Laughlin, Doug Lin, Norm Murray, Robert Noyes and Dimitar Sasselov for stimulating conversations.

\section{References}

Allende Prieto, \& Lambert, D. 1999 A\&A, 352, 555

Bryden, G., Chen, X., Lin, D. N. C., Nelson, \& R. P., Papaloizou, J. C. B. 1999, ApJ, 514, 344

Butler, R. P., Vogt, S. S., Marcy, G. W., Fischer, D. A., Henry, G. W., \& Apps, K. 2000, ApJ, 545, 504

Butler, R. P., Marcy, G. W., Fischer, D. A., Brown, T., Contos, A., Korzennik, S., Nisenson, P., \& Noyes, R.W. 1999, ApJ, 526, 916

Butler, R. P., Marcy, G. W., Williams, E., McCarthy, C., Dosanjh, P., \& Vogt, S. S. 1996, PASP, 108, 500

Cayrel de Strobel, G., Soubiran, C., \& Ralite, N. 2001, A\&A, 373, 159

Chiang, E. I., Fischer, D. A. \& Thommes, E. 2002, ApJ, 564, L105

Ford, E. B., Havlickova, M., Rasio, F.A., \& Yu, K. 2003, "Chaotic Interactions Among Multiple Planet Systems" to appear in Planetary Systems 8 Planets in Systems, Space Sciences Series of ISSI, Vol. 19, \& Space Sciences Reviews eds. Udry, S., Benz, W., \& von Steiger, R.

Fuhrmann, K. 1998, A\&A338, 161

Goldreich, P. \& Tremaine, S. 1980, ApJ, 241, 425

Gonzalez, G. 1997, MNRAS, 285, 403

Kupka F., Piskunov N.E., Ryabchikova T.A., Stempels H.C., \& Weiss W.W. 1999, A\&AS, 138, 119

Laughlin, G. P., \& Adams, F. C. 1997, ApJ, 491, 51

Laughlin, G. P. 2000, ApJ, 545, 1064

Levison, H. F., Lissauer, J. J., \& Duncan, M. J. 1998, AJ, 116, p.1998.

Lin, D. N. C., Bodenheimer, P., \& Richardson, D. C. 1996, Nature, 380, 606 
Lin, D. N. C. 1997, in ASP Conf. Ser. 121, "Accretion Phenomena \& Related Outflows," ed. D. T. Wickramasinghe, G. Bicknell, \& L. Ferrario (IAU Colloq. 163; San Francisco: ASP), 321

Malhotra, R. 2002, ApJ, 575, L33

Murray, N. \& Holman, M. 2001, Nature 410, 773

Murray, N., \& Chaboyer, B. 2002, ApJ, 566, 442

Pinsonneault, M. H., DePoy, D. L., \& Coffee, M 2001 ApJ, 556, 59

Rasio, F., \& Ford E., 1996, Science, 274, 954

Santos, N. C., Israelian, G. \& Mayor, M. 2000, A\&A, 363, 228

Santos, N. C., Israelian, G., Mayor, M., Rebolo, R., \& Udry, S. 2003. A\&A, 398,363

Valenti, J., \& Piskonov, N. 1996 A\&AS, 118, 595

Valenti, J., \& Fischer, D. 2004 ApJ, in press

Wallace, L., Hinkle, K., \& Livingston, W. 1998, An Atlas of the Spectrum of the Solar Photosphere from 13,500 to $28,000 \mathrm{~cm}^{-1}$ (3570 to $7405 \AA$ ) (Tucson: NOAO)

Weidenschilling, S. \& Marzari, F. 1996, Nature, 384, 619 